\title{
Evaluation of Symptoms in Cases of Otitis Media - A Clinical Study
}

\author{
Prasun Kumar Chattopadhyay ${ }^{1}$ \\ ${ }^{1}$ Senior Resident, Department of ENT, GMC Amritsar, Punjab, India.
}

\section{Abstract}

Background: Otitis media $(\mathrm{OM})$ is a group of inflammatory diseases of the middle ear. The present study was conducted to assess the cases of Otitis media. Subjects and Methods: The present study was conducted on 112 patients of OM of both genders. The case history and general physical examination was performed. General symptoms and types of OM were evaluated. Results: Out of 112 patients, males were 52 and females were 60. Acute OM was seen in 30 males and 32 females and chronic OM was seen in 22 males and 28 females. Common symptoms were cold seen in 68 , retro-auricular pain in 17 , fever in 80 , hearing impairment in 52 , ear discharge in 72 , tinnitus in 8 , vertigo in 45 and facial asymmetry in 26. Conclusion: Authors suggested that Otitis media is a common ear problem. They observed higher female prevalence than males.

Keywords: Middle ear, Otitis media, Tinnitus.

Corresponding Author: Dr. Prasun Kumar Chattopadhyay, House no 2132 urban estate phase 2, Patiala, 147001, Punjab, India.

Received: April 2019

Accepted: May 2019

\section{Introduction}

Otitis media is a group of inflammatory diseases of the middle ear. Occasionally a feeling of fullness is described. It is defined as the presence of non-infectious fluid in the middle ear for more than three months. Chronic suppurative otitis media (CSOM) is middle ear inflammation of greater than two weeks that results in episodes of discharge from the ear. It may be a complication of acute otitis media. Pain is rarely present. All three may be associated with hearing loss. The hearing loss in OME, due to its chronic nature, may affect a child's ability to learn. ${ }^{[1]}$

AOM is an infection of abrupt onset that usually presents with ear pain. In young children this may result in pulling at the ear, increased crying, and poor sleep. Decreased eating and a fever may also be present. Acute Otitis Media is one of the commonest infections of the mucosal lining of middle ear cleft. Adults make up less than $20 \%$ of patients presenting with acute otitis media. ${ }^{[2]}$

CSOM is also called chronic active mucosal otitis media, chronic oto-mastoiditis, and chronic tympanomastoiditis. A subset of CSOM may have cholesteatomas or other suppurative complications. ${ }^{[3]}$ The non-CSOM group includes such entities as chronic non-suppurative otitis media, chronic otitis media with effusion (COME), chronic secretory otitis media, chronic seromucous otitis media, chronic middle ear catarrh, chronic serous otitis media, chronic mucoid otitis media, otitis media with persistent effusions, and glue ear. All these are recurrent or persistent effusions in the middle ear behind an intact tympanic membrane in which the principal symptom, if present at all, is deafness and not ear discharge. ${ }^{[4]}$ The present study was conducted to assess the cases of otitis media.

\section{Subjects and Methods}

The present study was conducted in the department of ENT. It comprised of 112 patients of OM of both genders. All were informed regarding the study and written consent was obtained. Ethical clearance was taken from institutional ethical committee.

General information such as name, age, gender etc was noted. The case history and general physical examination was performed. General symptoms and types of OM were also evaluated. Results were subjected to statistical analysis. $P$ value $<0.05$ was considered significant.

\section{Results}

Table 1: Distribution of patients

\begin{tabular}{|l|l|l|}
\hline Gender & Male & Female \\
\hline Number & 52 & 60 \\
\hline
\end{tabular}

[Table 1] shows that out of 112 patients, males were 52 and females were 60 .

Table 2: Types of OM in both genders
\begin{tabular}{|l|l|l|}
\hline Types & Male & Female \\
\hline Acute OM & 30 & 32 \\
\hline Chronic OM & 22 & 28 \\
\hline
\end{tabular}

[Table 2] shows that acute OM was seen in 30 males and 32 females and chronic OM was seen in 22 males and 28 females. 


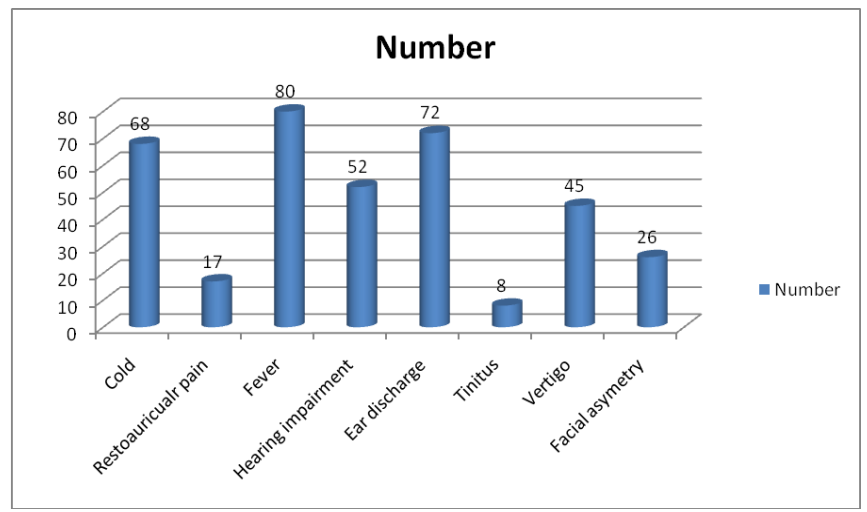

Figure 1: Symptoms of otitis media

[Figure 1] shows that common symptoms were cold seen in 68 , retro-auricular pain in 17 , fever in 80 , hearing impairment in 52 , ear discharge in 72 , tinnitus in 8 , vertigo in 45 and facial asymmetry in 26.

\section{Discussion}

The prevalence of chronic otitis media in the African countries are markedly ranging from $0.4 \%$ to $4.2 \%$. Perforation rates ranged from $0.4 \%$ to $2.8 \%$, and otorrhoea rates ranged from $0.4 \%$ to $3.6 \%$. Thus the prevalence of CSOM in Sub-Saharan Africa ranges from $0.4 \%$ to $4.2 \%$ regardless of definition. ${ }^{[5]}$

History-taking should be carried out to elicit the symptoms of ear pain, ear discharge, ear tugging or crying when the ear is touched, all of which suggest an ear problem. A history of previous ear discharge, especially when accompanied by episodes of colds, sore throat, cough or some other symptom of upper respiratory infection, should raise the suspicion of CSOM. A history of vigorous ear cleaning, itching or swimming that could traumatize the external ear canal suggests acute otitis externa (AOE), and not usually CSOM. ${ }^{[6]}$ A history of ear pain suggests AOE or AOM, not usually CSOM. In the case of AOM, the ear is only painful until the eardrum perforates, relieving the pressure. Thus, if the main symptom is painless otorrhoea, the duration of otorrhoea will help distinguish AOM from CSOM $^{[7]}$ The present study was conducted to assess the cases of otitis media.

In present study, out of 112 patients, males were 52 and females were 60 . Acute $\mathrm{OM}$ was seen in 30 males and 32 females and chronic OM was seen in 22 males and 28 females. Marchant et al8 in their study found that age group 20-30 years had maximum patients (males- 10 , females- 24 ) followed by 30-40 years (males- 8 , females- 11), 40-50 years (males- 7, females- 8), 50-60 years (males- 3, females- 6) and 60-70 years (males- 2, females- 5). Common symptoms were cold (30), fever (10), earache (66), hearing impairment (22), ear discharge (61), tinnitus (7), restoauricular pain (4), vertigo (2) and facial asymmetry (1). Common signs such as intact TM (55), bulging TM (20), discharge in external auditory canal (22), congested
TM (45), perforated TM (38), deviated nasal septum (33), mastoid tenderness (5) and facial nerve paralysis (2).

We found that common symptoms were cold seen in 68 , retro-auricular pain in 17 , fever in 80 , hearing impairment in 52, ear discharge in 72, tinnitus in 8 , vertigo in 45 and facial asymmetry in 26. Mukta et al, ${ }^{[9]}$ found that earache was the commonest symptom present in 65 patients. Spontaneous perforation was present in 37 patients. Earache, ear discharge \& hearing loss are the commonest symptoms in adults \& rate of spontaneous perforation is higher compared to children.

De Zinis et $\mathrm{al}^{[10]}$ reported acute mastoiditis as the commonest complication at $83 \%$ followed by facial nerve paralysis at $30 \%$. They also stated that full recovery of the facial nerve is to be expected irrespective of the management. The complications of AOM observed in the present study were ipsilateral lower motor neuron facial nerve paralysis ( 1 case), two patients had acute mastoiditis $\&$ one had labyrinthitis. All the patients with complications recovered with medical treatment. The patient with labyrinthitis had residual mixed hearing loss. The shortcoming of the study is small sample size. Even management of cases was also not compared.

\section{Conclusion}

Authors suggested that Otitis media is a common ear problem. They observed higher female prevalence than males.

\section{References}

1. Del Mar C, Glasziou P, Havem M. Are antibiotics indicated as initial treatment for children with acute otitis media? A meta-analysis. BMJ 1997; 314:1526.

2. Marchant CD, McMillan PM, Shurin PA. Objective diagnosis of otitis media in early infancy by tympanometry and ipsilateral acoustic reflex thresholds. J Pediatr 1986; 109: 590-595.

3. Van zuijlen, DiederickA, Schilder, Anne G.M et al. National differences in incidence of acute mastoiditis: relationship to prescribing patterns of antibiotics for acute otitis media? Paediatric Infectious Disease Journal 2001; 20: 140-144.

4. J Pukander. Clinical Features of Acute Otitis Media Among Children. ActaOto-laryngologica 1983, Vol. 95, No. 1-4, Pages 117-122.

5. Rea P, Graham J. Acute otitis media in children. Gleeson M. Scott Brown's otorhinolaryngology, Head \& Neck Surgery 7th ed. Hodder Arnold; 2008. 921-922.

6. Feingold M, Klein JO, Haslam GE, et al. Acute otitis media in children. Am J Dis Child 1966; 111: 361-365.

7. Coffey JD. Otitis media in the practice of pediatrics: bacteriological and clinical observations. Pediatrics 1966; 38: 25-32.

8. Marchant CD, McMillan PM, Shurin PA. Objective diagnosis of otitis media by tympanometry and ipsilateral acoustic reflex thresholds. J Pediatr 1986; 109: 590-595.

9. MuktaPagrani, AbhinavSrivastava, Chander Mohan. Adult Onset Acute Otitis Media - A Preliminary Report. Journal of Evolution of Medical and Dental Sciences 2014; 3: 5094-5099.

10. De Zinis R, Luca O, Gamba P, Balzanelle C. Acute Otitis Media And Facial Nerve Paralysis in Adults. Otology and otoneurology 2003; 24:113-7. 
Copyright: ( ) the author(s), 2019. It is an open-access article distributed under the terms of the Creative Commons Attribution License (CC BY 4.0), which permits authors to retain ownership of the copyright for their content, and allow anyone to download, reuse, reprint, modify, distribute and/or copy the content as long as the original authors and source are cited.

How to cite this article: Chattopadhyay PK. Evaluation of Symptoms in Cases of Otitis Media - A Clinical Study. Asian J. Med. Res. 2019;8(2):EN01-EN03.

DOI: dx.doi.org/10.21276/ajmr.2019.8.2.EN1

Source of Support: Nil, Conflict of Interest: None declared. 